\title{
Context computing for internet of things
}

\author{
Hector John T. Manaligod ${ }^{1}$ Michael Joseph S. Diño ${ }^{2}$ Supratip Ghose ${ }^{3} \cdot J_{\text {Jungsoo Han }}^{4}$
}

Published online: 14 November 2019

(c) Springer-Verlag GmbH Germany, part of Springer Nature 2019

Context-aware computing is an ambient-intelligence environment for adapting to the situations around humans, to their surroundings, and their use of software and hardware. Through IoT-based sensing, context-aware computing connects a variety of information found in the real world to ambient intelligence. A variety of IoT devices, such as smartphones, tablet PCs, wearable devices, smart bands, smart sensors, cameras, microphones, and GPS devices, can be connected with each other to collect context-aware data of the user's surroundings in real time. Ambient intelligence turns real situations into information, and provides a user-friendly intelligence service using the information. The information from a real situation makes it possible to realize human-oriented decision making through the application of a variety of machine learning techniques, such as feature extraction, learning, and inference. Along with the information collected in real-time, from the underlying data such as user preferences or behavior pattern data are analyzed and learned to achieve adaptive decision-making in consideration of personal situations. The main issues of context-aware

Jungsoo Han

jungsoo.han.k@gmail.com

Hector John T. Manaligod

hjmanaligod@gmail.com

Michael Joseph S. Diño

mjdino@fatima.edu.ph

Supratip Ghose

sgresearch@gmail.com

1 Computer Applications Department, School of Management and Information Technology, De La Salle College of St. Benilde, Manila, Philippines

2 Research Development \& Innovation Center, Our Lady of Fatima University, Valenzuela, Philippines

3 Department of Computer Science and Engineering, University of Information Technology \& Sciences, Dhaka, Bangladesh

4 Division of Information \& Communication, Baekseok University, 76, Munam-ro, Anseo-dong, Dongnam-gu, Cheonan-Si, Chungcheongnam-do, South Korea computing are the integration of the data collected from multiple data sources and the protection of personal information about the end-users. To address these issues, various studies have been conducted in the computer science area. The central issue is to introduce selected research papers which includes trends in topics like context computing for the internet of things, context computing for networks, ambient embedded systems, context software, ambient context computing, adaptive knowledge base systems, advancement in wireless technologies, ambient IoT contexts, hybrid networking systems, artificial intelligence, innovative applications of semantic computing, knowledge mining, big data analysis, and ambient intelligence.

The first paper by Hakim et al. (2018) suggests a nonintrusive contextual dynamic reconfiguration process of an ambient-intelligence IoT system. They propose contextual dynamic reconstruction using an ambient architecture-level IoT framework with more flexibility and comfort of use. This study focuses on reconfiguring IoT systems using an evolution manager's structure, and focuses on reconfiguration steps using processing-context data and a decision-making process. In addition, it develops a smart home implementation based on ambient devices and a home gateway using the autonomic computing MAPE/K loop and the cisco packet tracer simulator. It monitors the progress of context data, analyzing, planning, and executing contextual dynamic reconfigurations. The second paper by Kim et al. (2018) develops a context-aware adaptive algorithm using ambient-intelligence, dynamic adaptive streaming over HTTP (DASH) in mobile edge computing (MEC). The developed algorithm aims to select the optimal streaming segment, and reduces network latency in adaptive MEC streaming. This study applies an MLP deep learning algorithm and the multilayer perceptron classifier in the intermediate roles between a core server and a client application. It guarantees high quality of service as well as consistent network connections by improving quality streaming and reducing network latency.

The third paper by Chung et al. (2018) proposes ambient context modeling for the health-risk assessment using a 
knowledge base and deep neural networks. This study aims to generate a deep neural-network learning model with a knowledge base for ambient context-awareness. It uses entropy-based relevance feedback from the context patterns extracted from personal health record (PHR), electronic medical record (EMR), open-access directory (OAD), and phased history data (PHD) extraction. In addition, it analyzes an association of hidden contexts using an ontology inference engine and ambient-context pattern mining of structured and unstructured contexts. The developing ambient intelligence (AMI) architecture has health care technology that can provide health-risk prediction services to a patient with a disease. The fourth paper by Jin et al. (2018) develops the influencing factors of an information-seeking behavior in an ambient-intelligence context. The developed model focuses on a mediating role in the influence of ambient IoT trust and perceived-risk factors for an information-seeking ability and an information-seeking motive. It explores the context-aware mechanism between the characteristics of influencing factors and information-seeking behavior in combination with the context of ambient intelligence. In addition, the authors focus on reliability analysis, validity analysis of exploratory factors and confirmatory factors, model evaluations, and hypothesis testing.

The fifth paper by Park et al. (2018) introduces an ambient-intelligence context aware-based intrusion detection system (IDS) using machine learning for a smart factory. The suggested ambient context-aware objects are shared in a real-time context in an ambient context-aware environment and with smart objects with an ambient sensor in various spaces. The developing context aware-based IDS provides an effective detection rate for possible intrusion scoring in hybrid unsupervised and supervised learning methods. In addition, it uses drill-down analysis models with an integrated time and data standard. The developing architecture consists of data capture and a parsing phase, an inference and model building phase, and a threat visualization phase. It detects context awareness, and makes decisions through secure 3D analysis using machine learning. The sixth paper by Liu et al. (2018) proposes examining the effects of context awareness on ambient intelligence of logistics-information quality using awareness compatibility as a moderator variable. The study focuses on context awareness-based AMI and predicts intentions according to the context-awareness information. Then, it is applied to logistics services with real-time ambient-intelligence context awareness through mobile platforms. It provides sufficient information quality and logistics risk reductions during the decision-making process. This scheme aims to generate personalization, pervasiveness, contextuality, and interactivity for positive and negative effects on logistics-information quality.

The seventh paper by Jang et al. (2019) suggests narrative, context-based, data-to-text generation in natural language generation models of ambient intelligence. The composition of the data-to-text generation model has sentence representations with similar meanings from text generation, and a corpus from structured and unstructured contexts. Besides, it has coherence and offers a diversity of information using a copy mechanism and a narrative context. Sentence representation has sentence embedding and sentence clustering to learn sentences as vectors, with vector similarities from a large corpus. It consists of surface realization and narrative context planning using an attention-based recurrent neural network (RNN) model for overcoming the sparsity of sentence patterns from ambient-intelligence IoT devices. The eighth paper by Khan and Kumar (2019) develops an ambient crop field monitoring service modified with information from an ambient sensor. It improves ambient context-based agricultural monitoring using a mobile sink (MS) from the quality-of-service parameters in wireless sensor networks (WSNs). This study aims to design an improved path using an ambient sensor node for mobile sink-travel paths and a mobile sink tree (MST) in an ambient context-based agricultural WSN. It advances diamond-shape MS moving strategies for improving ambient sensor network performance. WSNs have a mobile sink path and an ambient context collected by a context-awareness model. It improves the context-based agricultural WSN performance using an ambient crop field-monitoring model.

The ninth paper by Yang et al. (2018) proposes a group preference-based similarity (GPS) model through a comparison of clustering techniques and context-aware recommendation systems in ambient intelligence. This study develops the user preference model as a group preferencebased similarity model for top-K recommendations. This uses user- and group-preference models for tracing a thicker grasp of engagements and interactions. The suggested algorithm aims to generate a sequence of learning parameters using an adaptive Bayesian personalized ranking algorithm. It solves longstanding problems in cold start or in the data sparsity of real-world datasets, and offers the benefit of robust independence from matrix factorization of ambient interactions. The tenth paper by Kim and Chung (2018) suggests an adaptive context prediction model using neural networking on an ambient-intelligence context-mining life care platform. This study flexibly develops a context ontologybased machine-learning model using neural networking to generate health-risk factor coefficients in a knowledge base under IEEE 101073 and health level 7 (HL7). This model depicts an adaptive prediction model of contexts in ambient intelligence by calculating weight factor and the health factor similarity through a neural learning model. Moreover, it advances collaborative filtering predictions using decision-making and a convolutional neural network (CNN) for the classification of unstructured life care contexts. The eleventh paper by Kumar and Kamalraj (2019) proposes an 
ambient-intelligence architecture using a modified square root carry-select adder and a modified Russian peasant multiplier. The study aims to construct a half-band filter with a cascade structure for biomedical contexts, such as electroencephalograms (EEGs), electrocardiograms (ECGs), etc. In addition, this study focuses on magnetic resonance in porous media (MRPM)-based desensitized half-band finite impulse response (FIR) to improve biomedical applications with Gaussian noise signals in ambient intelligence.

We really appreciate the reviewers for their valuable contributions. We would also like to express thanks to Professor Vincenzo Loia who is the editor in chief of Journal of Ambient Intelligence and Humanized Computing.

\section{References}

Chung K, Yoo H, Choe D (2018) Ambient context-based modeling for health risk assessment using deep neural network. J Ambient Intell Humaniz Comput. https://doi.org/10.1007/s12652-018-1033-7

Hakim A, Amirat A, Oussalah MC (2018) Non-intrusive contextual dynamic reconfiguration of ambient intelligent IoT systems. J Ambient Intell Humaniz Comput. https://doi.org/10.1007/s1265 2-018-1127-2

Jang J, Noh H, Lee Y, Pantel SM, Rim H (2019) Narrative contextbased data-to-text generation for ambient intelligence. J Ambient Intell Humaniz Comput. https://doi.org/10.1007/s12652-01901176-7

Jin H, Wang H, Gong C, Liu L (2018) A study on the influencing factors of consumer information-seeking behavior in the context of ambient intelligence. J Ambient Intell Humaniz Comput. https:// doi.org/10.1007/s12652-018-1005-y

Khan THF, Kumar DS (2019) Ambient crop field monitoring for improving context based agricultural by mobile sink in WSN. J Ambient Intell Humaniz Comput. https://doi.org/10.1007/s1265 2-019-01177-6

Kim JC, Chung K (2018) Neural-network based adaptive context prediction model for ambient intelligence. J Ambient Intell Humaniz Comput. https://doi.org/10.1007/s12652-018-0972-3

Kim J, Won Y, Yoon C, Kim JY, Park S, Ryou JC, Ma LV (2018) A context-aware adaptive algorithm for ambient intelligence DASH at mobile edge computing. J Ambient Intell Humaniz Comput. https://doi.org/10.1007/s12652-018-1049-z

Kumar CU, Kamalraj S (2019) Ambient intelligence architecture of MRPM context based 12-tap further desensitized half band FIR filter for EEG signal. J Ambient Intell Humaniz Comput. https:// doi.org/10.1007/s12652-019-01237-x

Liu C, Park EM, Jiang F (2018) Examining effects of context-awareness on ambient intelligence of logistics service quality: user awareness compatibility as a moderator. J Ambient Intell Humaniz Comput. https://doi.org/10.1007/s12652-018-1004-z

Park ST, Li G, Hong JC (2018) A study on smart factory-based ambient intelligence context-aware intrusion detection system using machine learning. J Ambient Intell Humaniz Comput. https://doi. org/10.1007/s12652-018-0998-6

Yang Y, Hooshyar D, Jo J, Lim H (2018) A group preference-based item similarity model: comparison of clustering techniques in ambient and context-aware recommender systems. J Ambient Intell Humaniz Comput. https://doi.org/10.1007/s1265 2-018-1039-1

Publisher's Note Springer Nature remains neutral with regard to jurisdictional claims in published maps and institutional affiliations. 